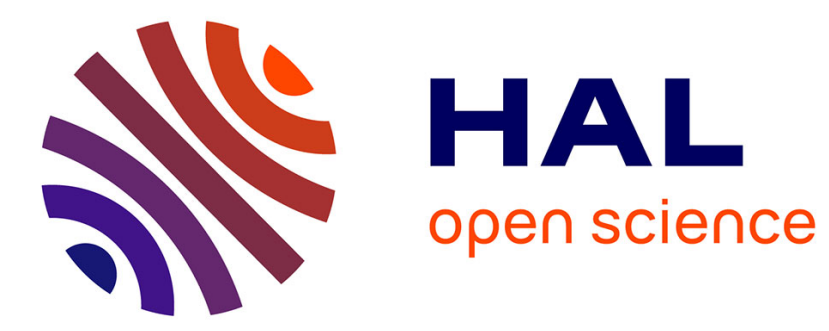

\title{
Models and Strategies for Variants of the Job Shop Scheduling Problem
}

\author{
Diarmuid Grimes, Emmanuel Hébrard
}

\section{To cite this version:}

Diarmuid Grimes, Emmanuel Hébrard. Models and Strategies for Variants of the Job Shop Scheduling Problem. Principles and Practice of Constraint Programming - CP 2011, Sep 2011, Perugia, Italy. pp.356-372, 10.1007/978-3-642-23786-7 . hal-00626799

\section{HAL Id: hal-00626799 \\ https://hal.science/hal-00626799}

Submitted on 27 Sep 2011

HAL is a multi-disciplinary open access archive for the deposit and dissemination of scientific research documents, whether they are published or not. The documents may come from teaching and research institutions in France or abroad, or from public or private research centers.
L'archive ouverte pluridisciplinaire HAL, est destinée au dépôt et à la diffusion de documents scientifiques de niveau recherche, publiés ou non, émanant des établissements d'enseignement et de recherche français ou étrangers, des laboratoires publics ou privés. 


\title{
Models and Strategies for Variants of the Job Shop Scheduling Problem
}

\author{
Diarmuid Grimes $^{1}$ and Emmanuel Hebrard ${ }^{2,3}$ \\ 1 Cork Constraint Computation Centre \\ University College Cork, Ireland \\ d.grimes@ 4c.ucc. ie \\ 2 CNRS ; LAAS ; 7 avenue du colonel Roche, F-31077 Toulouse, France \\ ${ }^{3}$ Université de Toulouse ; UPS, INSA, INP, ISAE ; UT1, UTM, LAAS ; F-31077 Toulouse, France \\ hebrardelaas.fr
}

\begin{abstract}
Recently, a variety of constraint programming and Boolean satisfiability approaches to scheduling problems have been introduced. They have in common the use of relatively simple propagation mechanisms and an adaptive way to focus on the most constrained part of the problem. In some cases, these methods compare favorably to more classical constraint programming methods relying on propagation algorithms for global unary or cumulative resource constraints and dedicated search heuristics. In particular, we described an approach that combines restarting, with a generic adaptive heuristic and solution guided branching on a simple model based on a decomposition of disjunctive constraints. In this paper, we introduce an adaptation of this technique for an important subclass of job shop scheduling problems (JSPs), where the objective function involves minimization of earliness/tardiness costs. We further show that our technique can be improved by adding domain specific information for one variant of the JSP (involving time lag constraints). In particular we introduce a dedicated greedy heuristic, and an improved model for the case where the maximal time lag is 0 (also referred to as no-wait JSPs).
\end{abstract}

\section{Introduction}

Scheduling problems come in a wide variety and it is natural to think that methods specifically engineered for each variant would have the best performance. However, it was recently shown this is not always true. Tamura et al. introduced an encoding of disjunctive and precedence constraints into conjunctive normal form formulae [22]. Thanks to this reformulation they were the first to report optimality proofs for all open shop scheduling instances from three widely studied benchmarks. Similarly the hybrid CP/SAT solver lazy-FD [10] was shown to be extremely effective on Resource-Constrained Project scheduling (RCPSP) [21].

Previously, we introduced an approach for open and job shop problems with a variety of extra constraints $[12,13]$ using simple reified binary disjunctive constraints combined with a number of generic SAT and AI techniques: weighted degree variable ordering [5], solution guided value ordering [3], geometric restarting [25] and nogood recording from restarts [15]. It appears that the weighted degree heuristic efficiently detects the most constrained parts of the problem, focusing search on a fraction of the variables.

The simplicity of this approach makes it easy to adapt to various constraints and objective functions. One type of objective function that has proven troublesome for traditional $\mathrm{CP}$ scheduling techniques involves minimizing the sum of earliness/tardiness costs, primarily due to the weak propagation of the sum objective [8]. In this paper we show how our basic JSP model can be adapted to handle this objective. Experimental results reveal that our approach is competitive with the state of the art on the standard benchmarks from the literature. 
Moreover, we introduce two refinements of our approach for problems with maximum time lags between consecutive tasks, where we incorporate domain specific information to boost performance. These time lag constraints, although conceptually very simple, change the nature of the problem dramatically. For instance, it is not trivial to find a feasible schedule even if we do not take into account any bound on the total makespan (unless scheduling jobs back to back). This has several negative consequences. Firstly, it is not possible to obtain a trivial upper bound of reasonable quality may be found by sequencing the tasks in some arbitrary order. The only obvious upper bound is to sequence the jobs consecutively. Secondly, since relaxing the makespan constraint is not sufficient to make the problem easy, our approach can have difficulty finding a feasible solution for large makespans, even though it is very effective when given a tighter upper bound. However because the initial upper bound is so poor, even an exploration by dichotomy of the objective variable's domain can take a long time.

We introduce a simple search strategy which, when given a large enough upper bound on the makespan, guarantees a limited amount of backtracking whilst still providing good quality solutions. This simple strategy, used as an initial step, greatly improves the performance of our algorithm on this problem type. We report several new best upper bounds and proofs of optimality on these benchmarks. Moreover, we introduce another improvement in the model of the particular case of No wait JSP where the tasks of each job must be directly consecutive. This variant has been widely studied, and efficient metaheuristics have been proposed recently. We report 5 new best upper bound, and close 9 new instances in standard data sets.

Finally, because there are few comparison methods in the literature for problems with strictly positive time lags, we adapted a job shop scheduling model written in Ilog Scheduler by Chris Beck [3], to handle time lag constraints. Our method outperforms this model when time lag constraints are tight (short lags), however when time lags are longer, the Ilog Scheduler model together with geometric restarts and solution guided search is better than our method.

\section{Background \& Previous work}

An $n \times m$ job shop problem (JSP) involves a set of $n m$ tasks $\mathcal{T}=\left\{t_{i} \mid 1 \leq i \leq n m\right\}$, partitioned into $n$ jobs $\mathcal{J}=\left\{J_{x} \mid 1 \leq x \leq n\right\}$, that need to be scheduled on $m$ machines $\mathcal{M}=\left\{M_{y} \mid 1 \leq y \leq m\right\}$. Each job $J_{x} \in \mathcal{J}$ is a set of $m$ tasks $J_{x}=\left\{t_{(x-1) * m+y} \mid 1 \leq y \leq m\right\}$. Conversely, each machine $M_{y} \in \mathcal{M}$ denotes a set of $n$ tasks (to run on this machine) such that: $\mathcal{T}=\left(\bigcup_{1 \leq x \leq n} J_{x}\right)=\left(\bigcup_{1 \leq y \leq m} M_{y}\right)$.

Each task $t_{i}$ has an associated duration, or processing time, $p_{i}$. A schedule is a mapping of tasks to time points consistent with sequencing and resource constraints. The former ensure that the tasks of each job run in a predefined order whilst the latter ensure that no two tasks run simultaneously on any given machine. In the rest of the paper, we shall identify each task $t_{i}$ with the variable standing for its start time in the schedule. We define the sequencing (2.1) and resource (2.2) constraints in Model 1.

Moreover, we shall consider two objective functions: total makespan, and weighted earliness/tardiness. In the former, we want to minimize the the total duration to run all tasks, that is, $C_{\text {max }}=\max _{t_{i} \in \mathcal{T}}\left(t_{i}+p_{i}\right)$ if we assume that we start at time 0 . In the latter, each job $J_{x} \in \mathcal{J}$ has a due date, $d_{x}$. There is a linear cost associated with completing a job before its due date, or the tardy completion of a job, with coefficient $w_{x}^{e}$ and $w_{x}^{t}$, respectively. (Note that these problems differ from Just in Time job shop scheduling problems[2], where each task has a due date.) If $t_{x m}$ is the last task of job $J_{x}$, then $t_{x m}+p_{x m}$ is its completion time, hence the cost of a job is then given by: $E T_{\text {sum }}=\sum_{J_{x} \in \mathcal{J}}\left(\max \left(w_{x}^{e}\left(d_{x}-t_{x m}-p_{x m}\right), w_{x}^{t}\left(t_{x m}+p_{x m}-d_{x}\right)\right)\right)$ 


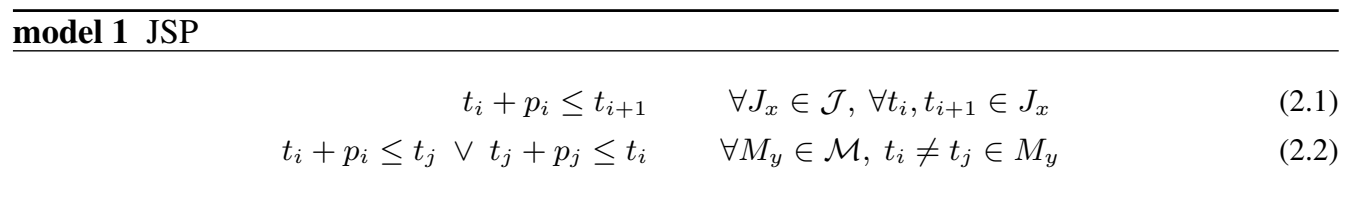

\subsection{Boolean Model}

In previous work [13] we described the following simple model for open shop and job shop scheduling. First, to each task, we associate a variable $t_{i}$ taking its value in $[0, \infty]$ that stands for its starting time. Then, for each pair of tasks sharing a machine we introduce a Boolean variable that stands for the relative order of these two tasks. More formally, for each machine $M_{y} \in \mathcal{M}$, and for each pair of tasks $t_{i}, t_{j} \in M_{y}$, we have a Boolean variable $b_{i j}$, and constraint (2.2) can be reformulated as follows:

$$
b_{i j}=\left\{\begin{array}{l}
0 \Leftrightarrow t_{i}+p_{i} \leq t_{j} \\
1 \Leftrightarrow t_{j}+p_{j} \leq t_{i}
\end{array} \quad \forall M_{y} \in \mathcal{M}, t_{i} \neq t_{j} \in M_{y}\right.
$$

Finally, the tasks of each job $J_{x}$, are kept in sequence with a set of simple precedence constraints $t_{i}+p_{i} \leq t_{i+1}$ for all $t_{i}, t_{i+1} \in J_{x}$.

For $n$ jobs and $m$ machines, this model therefore involves $n m(n-1) / 2$ Boolean variables, as many disjunctive constraints, and $n(m-1)$ precedence constraints. Bounds consistency (BC) is maintained on all constraints. Notice that state of the art CP models use instead $m$ global constraints to reason about unary resources. The best known algorithms for filtering unary resources constraints implement the edge finding, not-first/not-last, and detectable precedence rules with a $O(n \log n)$ time complexity [24]. One might therefore expect our model to be less efficient as $n$ grows. However, the quadratic number of constraints - and Boolean variables - required to model a resource in our approach has not proven problematic on the academic benchmarks tested on to date.

\subsection{Search Strategy}

We refer the reader to [12] for a more detailed description of the default search strategy used for job shop variants, and we give here only a brief overview.

This model does not involve any global constraint associated to a strong propagation algorithm. However, it appears that decomposing resource constraints into binary disjunctive elements is synergetic with adaptive heuristics, and in particular the weighted-degree-based heuristics [5]. (We note that the greater the minimum arity of constraints in a problem, the less discriminatory the weight-degree heuristic can be.) A constraint's weight is incremented by one each time the constraint causes a failure during search. This weight can then be projected on variables to inform the heuristic choices.

It is sufficient to decide the relative sequencing of the tasks, that is, the value of the Boolean variables standing for disjuncts. Because the domain size of these variables are all equal, we use a slightly modified version of the domain over weighted-degree heuristic, where weights and domain size are taken on the two tasks whose relative ordering is decided by the Boolean variable. Let $w\left(t_{i}\right)$ be the number of times search failed while propagating any constraint involving task $t_{i}$, and let $\min \left(t_{i}\right)$ and $\max \left(t_{i}\right)$ be, respectively, the minimum and maximum starting time of $t_{i}$ at any point during search. The next disjunct $b_{i j}$ to branch on is the one minimizing the value of:

$$
\left(\max \left(t_{i}\right)+\max \left(t_{j}\right)-\min \left(t_{i}\right)-\min \left(t_{j}\right)+2\right) /\left(w\left(t_{i}\right)+w\left(t_{j}\right)\right)
$$


A second important aspect is the use of restarts. It has been observed that weighted heuristics also have a good synergy with restarts [11]. Indeed, failures tend to happen at a given depth in the search tree, and therefore on constraints that often do not involve variables corresponding to the first few choices. As a result, early restarts will tend to favor diversification until enough weight has been given to a small set of variables, on which the search will then be focused. We use a geometric restarting strategy [25] with random tie-breaking. The geometric strategy is of the form $s, s r, s r^{2}, s r^{3}, \ldots$ where $s$ is the base and $r$ is the multiplicative factor. In our experiments the base was 256 failures and the multiplicative factor was 1.3. Moreover, after each restart, the dead ends of the previous explorations are stored as clausal nogoods [15].

A third very important feature is the idea of guiding search (branching choices) based on the best solution found so far. This idea is a simplified version of the solution guided approach (SGMPCS) proposed by Beck for JSPs [3]. Thus our search strategy can be viewed as variable ordering guided by past failures and value ordering guided by past successes.

Finally, before using a standard Branch \& Bound procedure, we first use a dichotomic search to reduce the gap between lower and upper bound. At each step of the dichotomic search, a satisfaction problem is solved, with a limit on the number of nodes.

\section{Job Shop with Earliness/Tardiness Objective}

In industrial applications, the length of the makespan is not always the preferred objective. An important alternative criterion is the minimization of the cost of a job finishing early/late. An example of a cost for early completion of a job would be storage costs incurred, while for late completion of a job these costs may represent the impact on customer satisfaction.

Although the only change to the problem is the objective function, our model requires a number of additional elements. When we minimize the sum of earliness and tardiness, we introduce $4 n$ additional variables. For each job $J_{x}$ we have a Boolean variable $e_{x}$ that takes the value 1 iff $J_{x}$ is finished early and the value 0 otherwise. In other words, $e_{x}$ is a reification of the precedence constraint $t_{x m}+p_{x m}<d_{x}$. Moreover, we also have a variable $E_{x}$ standing for the duration between the completion time of the last task of $J_{x}$ and the due date $d_{x}$ when $J_{x}$ is finished early: $E_{x}=e_{x}\left(d_{x}-t_{x m}-p_{x m}\right)$. Symmetrically, for each job $J_{x}$ we have Boolean variable $l_{x}$ taking the value 1 iff $J_{x}$ is finished late, and an integer variable $L_{x}$ standing for the delay (Model 2).

model 2 ET-JSP

$$
\begin{aligned}
\text { minimise } E T_{\text {sum }} \text { subject to : } & \\
E T_{\text {sum }}=\sum_{J_{x} \in \mathcal{J}}\left(w_{x}^{e} E_{x}+w_{x}^{t} L_{x}\right) & \\
e_{x} \Leftrightarrow\left(t_{x m}+p_{x m}<d_{x}\right) & \forall J_{x} \in \mathcal{J} \\
E_{x}=e_{x}\left(d_{x}-t_{x m}-p_{x m}\right) & \forall J_{x} \in \mathcal{J} \\
l_{x} \Leftrightarrow\left(t_{x m}+p_{x m}>d_{x}\right) & \forall J_{x} \in \mathcal{J} \\
L_{x}=l_{x}\left(t_{x m}+p_{x m}-d_{x}\right) & \forall J_{x} \in \mathcal{J}
\end{aligned}
$$

(constraints 2.1) \& (constraints 2.3)

Unlike the case where the objective involves minimizing the makespan, branching only on the disjuncts is not sufficient for these problems. Thus we also branch on the early and late 
Boolean variables, and on the variables standing for start times of the last tasks of each job. For these extra variables, we use the standard definition of domain over weighted degree.

\section{Job Shop Scheduling Problem with Time Lags}

Time lag constraints arise in many scheduling applications. For instance, in the steel industry, the time lag between the heating of a piece of steel and its moulding should be small [27]. Similarly when scheduling chemical reactions, the reactives often cannot be stored for a long period of time between two stages of a process to avoid interactions with external elements [19].

\subsection{Model}

The objective to minimise is represented by a variable $C_{\max }$ linked to the last task of each job by $n$ precedence constraints: $\forall x \in[1, \ldots, n] t_{x m}+p_{x m} \leq C_{\text {max }}$. The maximum time lag between two consecutive tasks is simply modelled by a precedence constraint with negative offset. Letting $L(i)$ be the maximum time lag between the tasks $t_{i}$ and $t_{i+1}$, we use the following model:

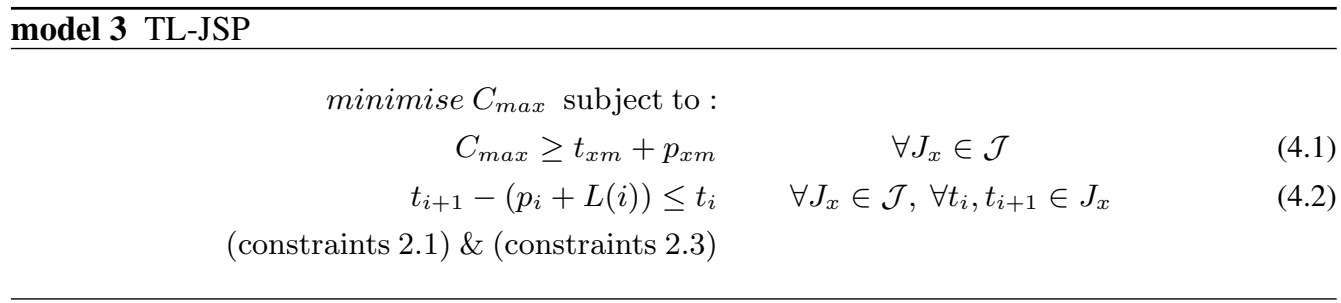

\subsection{Greedy Initialization}

In the classical job shop scheduling problem, one can consider tasks in any order compatible with the jobs and schedule them to their earliest possible start time. The resulting schedule may have a long makespan, however such a procedure usually produces reasonable upper bounds. With time lag constraints, however, scheduling early tasks of a job implicitly fixes the start times for later tasks, thus making the problem harder. Indeed, as soon as tasks have been fixed in several jobs, the problem becomes difficult even if there is no constraint on the length of the makepsan. Standard heuristics can thus have difficulty finding feasible solutions even when the makespan is not tightly bounded. In fact, we observed that this phenomenon is critical for our approach.

Once a relatively good upper bound is known our approach is efficient and is often able to find an optimal solution. However, when the upper bound is, for instance, the trivial sum of durations of all tasks, finding a feasible solution with such a relaxed makespan was in some cases difficult. For some large instances, no non-trivial solution was found, and on some instances of more moderate size, much computational effort was spent converging towards optimal values.

We therefore designed a search heuristic to find solutions of good quality, albeit very quickly. The main idea is to move to a new job only when all tasks of the same machine are completely sequenced between previous jobs. Another important factor is to make decisions based on the maximum completion time of a job, whilst leaving enough freedom within that job to potentially insert subsequent jobs instead of moving them to the back of the already scheduled jobs. 


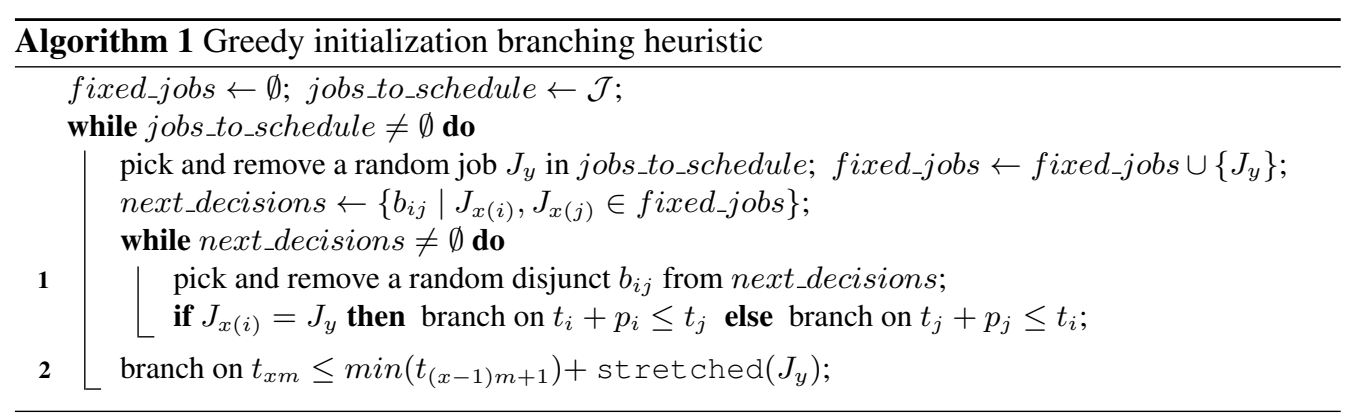

We give a pseudo-code for this strategy in Algorithm 1. The set jobs_to_schedule stands for the jobs for which sequencing is still open, whilst fixed_jobs contains the currently processed job, as well as all the jobs that are completely sequenced. On the first iteration of the outer "while" loop, a job is chosen. There is no disjunct satisfying the condition in Line 1, so this job's completion time is fixed to a value given by the stretched procedure (Line 2), that is, the minimum possible starting time of its first task, plus its total duration, plus the sum of the possible time lags.

On the second iteration and beyond, a new job is selected. We then branch on the sequencing decisions between this new job and the rest of the set fixed_jobs before moving to a new job. We call $J_{x(i)}$ the job that contains task $t_{i}$, and observe that for any unassigned Boolean variable $b_{i j}$, either $J_{x(i)}$ or $J_{x(j)} \in$ fixed_jobs must be the last chosen job $J_{y}$. The sequencing choice that sets a task of the new job before a task of previously explored jobs is preferred, i.e., considered in the left branch. Observe that a failure due to time lag constraints can be raised only in the inner "while" loop. Therefore, if the current upper bound on the makespan is large enough, this heuristic will ensure that we never backtrack on a decision on a task. We randomize this heuristic and use several iterations (1000 in the present set of experiments) to find a good initial solution.

\subsection{Special Case: Job Shop with no-wait problems}

The job shop problem with no-wait refers to the case where the maximum time-lag is set to 0 , i.e. each task of a job must start directly after its preceding task has finished. In this case one can view the tasks of the job as one block.

In [12] we introduced a simple improvement for the no-wait class based on the following observation: if no delay is allowed between any two consecutive tasks of a job, then the start time of every task is functionally dependent on the start time of any other task in the job. The tasks of each job can thus be viewed as one block. We therefore use a single variable $J_{x}$ standing for the starting times of the job of same name.

We call $J_{x(i)}$ the job of task $t_{i}$, and we define $h_{i}$ as the total duration of the tasks coming before task $t_{i}$ in its job $J_{x(i)}$. That is, $h_{i}=\sum_{k \in\left\{k \mid k<i \wedge t_{k} \in J_{x(i)}\right\}} p_{k}$. For every pair of tasks $t_{i} \in J_{x}, t_{j} \in J_{y}$ sharing a machine, we use the same Boolean variables to represent disjuncts as in the original model, however linked by the following constraints:

$$
b_{i j}=\left\{\begin{array}{l}
0 \Leftrightarrow J_{x}+h_{i}+p_{i}-h_{j} \leq J_{y} \\
1 \Leftrightarrow J_{y}+h_{j}+p_{j}-h_{i} \leq J_{x}
\end{array}\right.
$$

Although the variables and constants are different, these are the same constraints as used in the basic model. The no-wait JSP can therefore be reformulated as shown in Model 4, where the variables $J_{1}, \ldots, J_{n}$ represent the start time of the jobs and $f(i, j)=h_{i}+p_{i}-h_{j}$. 
model 4 NW-JSP

$$
\begin{gathered}
\begin{array}{c}
\text { minimise } C_{\max } \text { subject to : } \\
C_{\max } \geq J_{x}+\sum_{t_{i} \in J_{x}} p_{i} \\
b_{i j}=\left\{\begin{array}{l}
0 \Leftrightarrow J_{x(i)}+f(i, j) \leq J_{x(j)} \\
1 \Leftrightarrow J_{x(j)}+f(j, i) \leq J_{x(i)}
\end{array} \quad \forall M_{y} \in \mathcal{M}, t_{i} \neq t_{j} \in M_{y}\right.
\end{array}
\end{gathered}
$$

However, we can go one step further. For a given pair of jobs $J_{x}, J_{y}$ the set of disjunct between tasks of these jobs define as many conflict intervals for the start time of one job relative to the other. For two tasks $t_{i}$ and $t_{j}$, we have $\left.J_{x(j)} \notin\right] J_{x(i)}-f(j, i), J_{x(i)}+f(i, j)[$. However, these intervals may overlap or subsume each other. It is therefore possible to tighten this encoding by computing larger intervals, that we shall refer to as maximal forbidden intervals, hence resulting in fewer disjuncts. We first give an example, and then briefly describe a procedure to find maximal forbidden intervals.

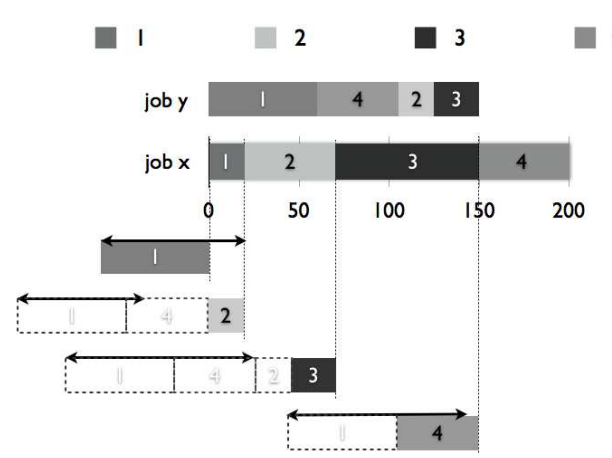

(a) Sample problem.

$\begin{array}{lcccc}\text { Machine } & 1 & 2 & 3 & 4 \\ t_{i}, t_{j} & t_{1}, t_{5} & t_{2}, t_{7} & t_{3}, t_{8} & t_{4}, t_{6} \\ p_{i} & 20 & 50 & 80 & 50 \\ h_{i} & 0 & 20 & 70 & 150 \\ p_{j} & 60 & 20 & 25 & 45 \\ h_{j} & 0 & 105 & 125 & 60 \\ -f(j, i) & -60 & -105 & -80 & 45 \\ f(i, j) & 20 & -35 & 25 & 140\end{array}$

(b) Values of $p, h$ and $f$.

Fig. 1: Computation of conflict intervals.

In Figure 1a we illustrate two jobs $J_{x}=\left\{t_{1}, t_{2}, t_{3}, t_{4}\right\}$ and $J_{y}=\left\{t_{5}, t_{6}, t_{7}, t_{8}\right\}$. The number and shades of grey stand for the machine required by each task. The length of the tasks are respectively $\{20,50,80,50\}$ for $J_{x}$ and $\{60,45,20,25\}$ for $J_{y}$. In Figure $1 \mathrm{~b}$ we give, for each machine, the pair of conflicting tasks, their durations and the corresponding forbidden intervals.

For each machine $M_{k}$, let $t_{i}$ be the task of $J_{x}$ and $t_{j}$ the task of $J_{y}$ that are both processed on machine $M_{k}$. Following the reasoning used in Model 4, we have a conflict interval (represented by black arrows in Figure 1a) for each pair of tasks sharing the same machine: $\left.J_{y} \notin\right] J_{x}-$ $f(j, i), J_{x}+f(i, j)\left[\right.$. In the example the forbidden intervals for $J_{y}$ are therefore: $] J_{x}-60, J_{x}+$ $20[\ldots] J_{x}-105, J_{x}-35[\ldots] J_{x}-80, J_{x}+25[\ldots] J_{x}+45, J_{x}+140[$. However, these intervals can be merged, yielding larger (maximal) forbidden intervals, in which case we have: $J_{y} \notin$ ] $J_{x}-105, J_{x}+25\left[\wedge J_{y} \notin\right] J_{x}+45, J_{x}+140[$.

Given two jobs $J_{x}$ and $J_{y}$, Algorithm 2 computes all maximal forbidden intervals efficiently (in $O(m \log m)$ steps). First, we build a list of pairs whose first element is an end point of a conflict interval, and second element is either +1 if it is the start, and -1 otherwise. Then these 


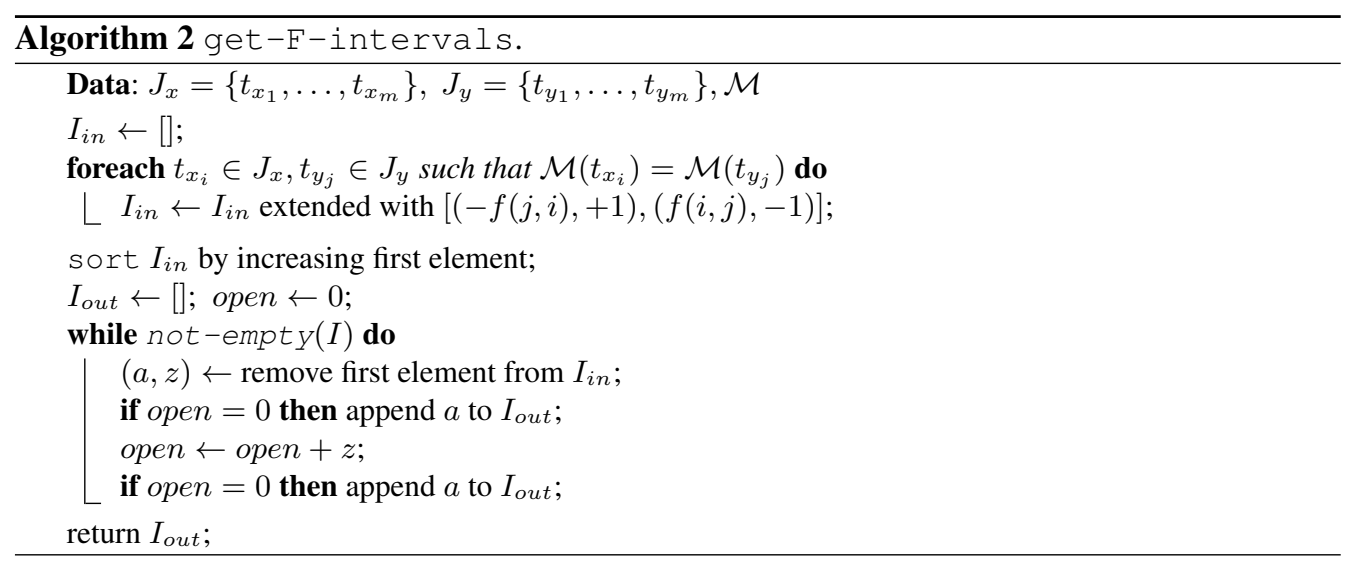

pairs are sorted by increasing first element. Now we can scan these pairs and count, thanks to the second element, how many intervals are simultaneously open. When we go from 0 to 1 open intervals, this marks the start of a maximal forbidden interval, and conversely the end when we go from 1 to 0 open intervals. The list $I_{\text {out }}$ has $2 k$ elements, and the $2 i+1^{\text {th }}$ and $2 i+2^{\text {th }}$ elements are read as the start and end of a forbidden interval.

Given this set of forbidden intervals, we can represent the conflicts between $J_{x}$ and $J_{y}$ with the following set of Boolean variables and disjunctive constraints:

$$
b_{x y}{ }^{105,25}=\left\{\begin{array}{c}
0 \Leftrightarrow J_{y}+105 \leq J_{x} \\
1 \Leftrightarrow J_{x}+25 \leq J_{y}
\end{array} \quad b_{x y}{ }^{45,140}=\left\{\begin{array}{c}
0 \Leftrightarrow J_{y}-45 \leq J_{x} \\
1 \Leftrightarrow J_{x}+140 \leq J_{y}
\end{array}\right.\right.
$$

In the previous encoding we would have needed 4 Boolean variables and as many disjunctive constraints (one for each pair of tasks sharing a machine). We believe, however, that the main benefit is not the reduction in size of the encoding. Rather, it is the tighter correlation between the model and the real structure of the problem which helps the heuristic to make good choices.

model 5 NW-JSP

minimise $C_{\max }$ subject to :

$$
\begin{gathered}
C_{\max } \geq J_{x}+\sum_{t_{i} \in J_{x}} p_{i} \\
b_{i j}{ }^{a, b}=\left\{\begin{array}{l}
0 \Leftrightarrow J_{y}-a \leq J_{x} \\
1 \Leftrightarrow J_{x}+b \leq J_{y}
\end{array} \quad \forall J_{x} \neq J_{y} \in \mathcal{J},[a, b] \in \operatorname{get}-\mathrm{F}-\text { intervals }\left(J_{x}, J_{y}, \mathcal{M}\right)(4.6)\right.
\end{gathered}
$$

\section{Experimental Evaluation}

The full experimental results, with statistics for each instance, as well as benchmarks and source code are online: http://homepages. laas.fr/ehebrard/jsp-experiment.html. 


\subsection{Job Shop with Earliness/Tardiness Objective}

The best complete methods for handling these types of problem are the CP/LP hybrid of Beck and Refalo [4] and the MIP approaches of Danna et al. [9], and Danna and Perron [8], while more recently Kebel and Hanzalek proposed a pure CP approach [14]. Danna and Perron also proposed an incomplete approach based on large neighborhood search [8].

Our experiments were run on an Intel Xeon $2.66 \mathrm{GHz}$ machine with $12 \mathrm{~GB}$ of ram on Fedora 9. Each algorithm run on a problem had an overall time limit of 3600s, and there were 10 runs per instance. We report our results in terms of the best and worst run. We tested our method on two benchmarks which have been widely studied in the literature. The comparison experimental results are taken from [9] and [8], where all experiments were performed on a $1.5 \mathrm{GHz}$ Pentium IV system running Linux. For the first benchmark, these algorithms had a time limit of 20 minutes per instance, while for the second benchmark the algorithms had a time limit of 2 hours.

The comparison methods are as follows:

- MIP: Default CPLEX in [9], run using a modified version of ILOG CPLEX 8.1

- CP: A pure constraint programming approach introduced by Beck and Refalo in [4], run using ILOG Scheduler 5.3 and ILOG Solver 5.3

- CRS-ALL: A CP/LP hybrid approach proposed by Beck and Refalo in [4], run using ILOG CPLEX 8.1, ILOG Hybrid 1.3.1, ILOG Scheduler 5.3 and ILOG Solver 5.3

- uLNS: An unstructured large neighborhood search MIP method proposed by Danna and Peron in [8], run using a modified version of ILOG CPLEX 8.1

- sLNS: A structured large neighborhood search CP/LP method proposed by Danna and Peron in [8], run using ILOG Scheduler 5.3, ILOG Solver 5.3 and ILOG CPLEX 8.1

The first benchmark consists of 9 sets of problems generated by Beck and Refalo [4] using the random JSP generator of Watson et al. [26]. For instance size $\mathcal{J} x \mathcal{M}$, there were three sets

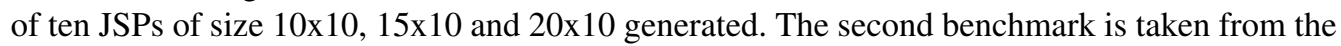
genetic algorithms (GA) literature and was proposed by Morton and Pentico [18]. There are 12 instances, with problem size ranging from $10 \times 3$ to $50 \times 8$. Jobs in these problems do have release dates. Furthermore earliness and tardiness costs of a job are equal.

We present results on the randomly generated ETJSPs in Table 1 in terms of number of problems solved to optimality and sum of the upper bounds, for each algorithm. ${ }^{4}$ Here, the column "Best" for our method means the number of problems solved to optimality on at least one of the ten runs on the instance, while the column "Worst" refers to the number of problems solved to optimality on all ten runs. We also report the mean cpu time in seconds for our method.

We first consider the number of problems solved to optimality (columns "Opt."). While there is little difference in the performance of our method and that of uLNS and CRS-ALL on the looser instances (looseness factor of 1.3 and 1.5), we see that our method is able to close three of the 23 open problems in the set with looseness factor 1.0. An obvious reason for this improvement with our method would be the difference in time limits and quality of machines. However, analysis of the results reveals that of the 68 problems solved to optimality on every run of our method, only 8 took longer than one second on average, and only one took longer than one minute (averaging 156s). Furthermore, uLNS only solved two problems to optimality when the time limit was increased to two hours [8]. Clearly our method is extremely efficient at proving optimality on these problems.

The previous results suggest that CRS-ALL is much better than uLNS on these problems. However, as was shown by Danna et al. [9], this may not be the case when the algorithms are

\footnotetext{
${ }^{4}$ Note that sLNS is not complete, hence it never proved optimality.
} 
Table 1: ET-JSP - Random Problems, Number Proven Optimal and Upper Bound Sum

\begin{tabular}{|c|c|c|c|c|c|c|c|c|}
\hline \multirow{2}{*}{ lf } & \multirow{2}{*}{ MIP } & \multirow{2}{*}{$\mathrm{CP}$} & \multirow{2}{*}{ uLNS } & \multirow{2}{*}{ sLNS } & \multirow{2}{*}{ CRS-All } & \multicolumn{3}{|c|}{ Model 2} \\
\hline & & & & & & Best & Worst & Avg. \\
\hline & $\sum u b$ & $\sum u b$ & $\sum \mathrm{ub}$ & $\sum u b$ & opt. $\quad \sum u b$ & opt. $\sum \mathrm{ub}$ & opt. $\sum u b$ & Time (s) \\
\hline 1.0 & $0 \quad 654,290$ & $0 \quad 1,060,634$ & $\begin{array}{ll}0 & 156,001\end{array}$ & 52,307 & 7885,546 & 1030,735 & $83 \overline{38,416}$ & 2534.86 \\
\hline 1.3 & $14 \quad 26,930$ & $61,248,618$ & $30 \quad 8,397$ & 8,397 & $30 \quad 8,397$ & $30 \quad 8,397$ & $30 \quad 8,397$ & 0.36 \\
\hline 1.5 & 7,891 & $61,672,511$ & 6,964 & 6,964 & 6,964 & 6,964 & 6,964 & 0.18 \\
\hline
\end{tabular}

Notes: Comparison results taken from [9], except uLNS, taken from [8].

Figures in bold are the best result over all methods.

compared based on the sum of the upper bounds found over the 30 "hard" instances (i.e. with looseness factor 1.0). In order to assess whether there was a similar deterioration in the performance of our method as for CRS-ALL on the problems where optimality was not proven, we report this data in the columns " $\sum$ ub" of Table 1.

We find, on the contrary, that the performance of our approach is even more impressive when algorithms are compared using this metric. The two large neighborhood search methods found the best upper bounds of the comparison algorithms with sLNS the most efficient by a factor of 2 over uLNS. However, there are a couple of points that should be noted here. Firstly sLNS is an incomplete method so cannot prove optimality, and secondly the sum of the worst upper bounds found by our method was still significantly better than that found by sLNS. Indeed, there was very little variation in performance for our method across runs, with an average difference of 256 between the best and worst upper bounds found.

Danna and Perron also provided the sum of the best upper bounds found on the hard instances over all methods they studied [8], which was 36,459 . This further underlines the quality of the performance of our method on these problems. Finally, we investigated the hypothesis that the different time limit and machines used for experiments could explain these results. We compared the upper bounds found by our method after the dichotomic search phase, where the maximum runtime of this phase over all runs per instance was 339s. The upper bound sums over the hard instances were 32,299 and 49,808 for best and worst respectively, which refutes this hypothesis.

Table 2 provides results on the second of the benchmarks (taken from the GA literature). Following the convention of previous work on these problems [23][4][9], we report the cost normalized by the weighted sum of the job processing times. We include the best results found by the GA algorithms as presented by Vázquez and Whitley [23]. We also provide an aggregated view of the results of each algorithm using the geometric mean ratio (GMR), which is the geometric mean of the ratio between the normalized upper bound found by the algorithm and the best known normalized upper bound, across a set of instances.

The performance of our method was less impressive for these problems, solving two fewer problems to optimality than uLNS, and achieving a worse GMR than either of the large neighborhood search methods. However, we remind the reader that all comparison methods had a 2 hour time limit on these instances, except the GA approaches for which the time limit was not reported. We further note that we find an improved solution for one instance (ljb10) and outperform all methods other than uLNS and sLNS. 
Table 2: ET-JSP - GA Problems, Normalized upper bounds

\begin{tabular}{|c|c|c|c|c|c|c|c|c|c|}
\hline \multirow{2}{*}{ Instance } & \multirow{2}{*}{ Size } & \multirow{2}{*}{ MIP } & \multirow{2}{*}{$\mathrm{CP}$} & \multirow{2}{*}{ uLNS } & \multirow{2}{*}{ sLNS } & \multirow{2}{*}{ CRS-All } & \multirow{2}{*}{$\begin{array}{l}\text { GA } \\
\text { Best }\end{array}$} & \multicolumn{2}{|c|}{ Model 2} \\
\hline & & & & & & & & Best & Worst \\
\hline jb1 & $10 \times 3$ & 0.191* & 0.474 & 0.191* & 0.191 & 0.191* & 0.474 & 0.191* & 0.191* \\
\hline jb2 & $10 \times 3$ & $0.137 *$ & 0.746 & $0.137 *$ & 0.137 & 0.531 & 0.499 & $0.137 *$ & $0.137 *$ \\
\hline jb4 & $10 \times 5$ & $0.568 *$ & 0.570 & $0.568 *$ & 0.568 & $0.568 *$ & 0.619 & $0.568 *$ & $0.568 *$ \\
\hline jb9 & $15 \times 3$ & 0.333* & 0.355 & $0.333^{*}$ & 0.333 & 1.216 & 0.369 & $0.333^{*}$ & 0.333* \\
\hline jb11 & $15 \times 5$ & 0.233 & 0.365 & $0.213^{*}$ & 0.213 & $0.213 *$ & 0.262 & 0.221 & 0.235 \\
\hline \multirow[t]{2}{*}{ jb12 } & $15 \times 5$ & $0.190 *$ & 0.239 & $0.190 *$ & 0.190 & $0.190 *$ & 0.246 & $0.190 *$ & $0.190 *$ \\
\hline & GMR & 1.015 & 1.774 & 1 & 1 & 1.555 & 1.610 & 1.006 & 1.017 \\
\hline ljb1 & 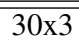 & $0.215 *$ & 0.847 & 0.215* & 0.215 & 0.295 & $\overline{0.279}$ & $\overline{0.215}$ & 0.221 \\
\hline ljb2 & $30 \times 3$ & 0.622 & 1.268 & 0.508 & 0.508 & 1.364 & 0.598 & 0.590 & 0.728 \\
\hline ljb7 & $50 \times 5$ & 0.317 & 0.614 & 0.123 & 0.110 & 0.951 & 0.246 & 0.166 & 0.256 \\
\hline ljb9 & $50 \times 5$ & 1.373 & 1.737 & 1.270 & 1.015 & 2.571 & 0.739 & 1.157 & 1.513 \\
\hline ljb10 & $50 \times 8$ & 0.820 & 1.569 & 0.558 & 0.525 & 1.779 & 0.512 & 0.499 & 0.637 \\
\hline \multirow[t]{2}{*}{ ljb12 } & $50 \times 8$ & 1.025 & 1.368 & 0.488 & 0.605 & 1.601 & 0.399 & 0.537 & 0.623 \\
\hline & GMR & 1.943 & 3.233 & 1.213 & 1.170 & 4.098 & 1.220 & 1.299 & 1.686 \\
\hline \multicolumn{2}{|c|}{ O"Overall GMR } & 1.329 & $\overline{2.434}$ & $\overline{c 1.084}$ & $\overline{1.068}$ & 2.305 & $\overline{1.408}$ & $\overline{c 1.118}$ & 1.256 \\
\hline
\end{tabular}

Comparison results taken from [9]. Figures in bold indicate best upper bound

found over the different algorithms. "*” indicates optimality was proven by the algorithm.

\subsection{Job Shop Scheduling Problem with positive Time Lags}

These experiments were run using the same settings as in Section 5.1. However, because of the large number of instances and algorithms, we used only 5 random runs per instance.

There are relatively few results reported for benchmarks with positive maximum time lag constraints, as most publications focus on the "no wait" case. Caumond et al. introduced a genetic algorithm [7]. Then, Artigues et al. introduced a Branch \& Bound procedure that allowed them to find lower bounds of good quality [1]. Therefore, in order to get a better idea of the efficiency of our approach, we adapted a model written by Chris Beck for Ilog Scheduler (version 6.3) to problems featuring time lag constraints. This model was used to showcase the SGMPCS algorithm [3]. We used the following two strategies: In the first, the next pair of tasks to schedule is chosen following the Texture heuristic/goal predefined in Ilog Scheduler and restarts following the Luby sequence [16] are performed, this was one of the default strategies used as a reference point in [3]. In the second, branching decisions are selected with the same "goal", however the previous best solution is used to decide wich branch should be explored first, and geometric restarts [25] are performed, instead of the Luby sequence. In other words, this is SGMPCS with a singleton elite solution. We denote the first method Texture-Luby and the second method Texture-Geom+Guided. These two methods were run on the same hardware with the same time limit and number of random runs as our method. Finally, we report results for our approach without the greedy initialization heuristic (Algorithm 1) in order to evaluate its importance.

We used the benchmarks generated by Caumond et al. in [7] by adding maximal time lag constraints to the Lawrence JSP instances of the OR-library ${ }^{5}$. Given a job shop instance N, and two parameters $x$ and $y$, a new instance $N_{-} \_x \_y$ is produced. For each job all maximal time lags

\footnotetext{
${ }^{5}$ http://people.brunel.ac.uk/ mastjjb/jeb/info.html
} 
are given the value $y m$, where $m$ is the average processing time over tasks of this job. The first parameter $x$ corresponds to minimal time lags and will always be 0 in this paper.

Table 3: TL-JSP - Comparison with related work (Time \& Upper bound).

\begin{tabular}{|c|c|c|c|c|c|c|}
\hline \multirow{2}{*}{ Instance } & \multicolumn{2}{|c|}{ [AHL] } & \multicolumn{2}{|c|}{$\overline{\text { [CLT] }}$} & \multicolumn{2}{|c|}{ Model 3} \\
\hline & time (s) & $C_{\max }$ & time (s) & $C_{\max }$ & time (s) & $C_{\max }$ \\
\hline $1 a 06 \_0 \_10$ & 707.00 & 927 & 0.00 & 926 & 0.03 & 926 \\
\hline $1 \mathrm{a} 06 \sigma_{0} 0_{-} 1$ & 524.00 & 1391 & 1839.00 & 1086 & 70.60 & 926 \\
\hline la07_0_10 & 518.00 & 1123 & 25.00 & 890 & 3600.00 & 890 \\
\hline la07_0_1 & 754.00 & 1065 & 1914.00 & 1032 & 3600.00 & 896 \\
\hline lao & 260.00 & 863 & 2.00 & 863 & 0.07 & 863 \\
\hline la08_0_1 & 587.00 & 1052 & 1833.00 & 1048 & 615.80 & 892 \\
\hline average & 558.33 & 1070 & 935.50 & 974 & 1314.41 & 898 \\
\hline$P R D$ & & 18.88 & & 8.32 & & 0.00 \\
\hline
\end{tabular}

Due to space limitations, we present most of our results in terms of each solver's average percentage relative deviation (PRD) given by the following formula: $P R D=\left(\left(C_{A l g}-\right.\right.$ $\left.\left.C_{R e f}\right) / C_{R e f}\right) * 100$, where $C_{A l g}$ is the best makespan found by the algorithm and $C_{R e f}$ is the best upper bound among all considered algorithms ${ }^{6}$. In Table 3, we first report a comparison with the genetic algorithm described in [7], denoted [CLT] and the adhoc Branch \& Bound algorithm introduced in [1], denoted [AHL]. We used only instances for which results were reported in both papers, and where the time lags were strictly positive, hence the relatively small data set. Despite that, and despite the difference in hardware and time limit, it is quite clear that our approach outperforms both the complete and heuristic methods on these benchmarks.

Table 4: TL-JSP - Comparison with Ilog Scheduler (Proofs of optimality \& Upper bound PRD).

\begin{tabular}{|c|c|c|c|c|c|c|c|c|}
\hline \multirow{3}{*}{ Instance Sets } & \multicolumn{4}{|c|}{ Texture } & \multicolumn{4}{|c|}{ Model 3} \\
\hline & \multicolumn{2}{|c|}{ Luby } & \multicolumn{2}{|c|}{ Geom+Guided } & \multicolumn{2}{|c|}{ no init. } & \multicolumn{2}{|c|}{ init. heuristic } \\
\hline & Opt. & $P R D$ & Opt. & $P R D$ & Opt. & $P R D$ & Opt. & $P R D$ \\
\hline $\operatorname{la}[1,40]]_{2} 0_{-}$ & 0.12 & 25.37 & 0.12 & 16.15 & 0.37 & 10.42 & 0.35 & 0.06 \\
\hline $\operatorname{la}[1,40], 0 \_0.25$ & 0.20 & 22.98 & 0.25 & 12.01 & 0.37 & 3.46 & 0.40 & 0.00 \\
\hline $1 \mathrm{a}[1,40]{ }_{-} 0_{-} 0.5$ & 0.22 & 19.47 & 0.25 & 5.17 & 0.37 & 2.62 & 0.42 & 0.00 \\
\hline $1 \mathrm{a}[1,40]]_{0}{ }_{1}$ & 0.35 & 15.76 & 0.42 & 1.18 & 0.40 & 17.43 & 0.45 & 0.47 \\
\hline $1 \mathrm{a}[1,40]{ }_{-}{ }_{2} 2$ & 0.67 & 7.35 & 0.75 & 0.13 & 0.67 & 74.16 & 0.70 & 0.37 \\
\hline $1 \mathrm{a}[1,40] \_0 \_3$ & 0.75 & 3.47 & 0.92 & 0.00 & 0.75 & 95.91 & 0.77 & 0.29 \\
\hline $1 \mathrm{a}[1,40]{ }_{0}{ }_{-10}$ & 0.95 & 0.10 & 0.97 & 0.00 & 0.92 & 0.04 & 0.92 & 0.05 \\
\hline
\end{tabular}

Next, in Table 4, we report results on all modified Lawrence instances for both Ilog Scheduler models, and the two version of Model 3, with and without the greedy initialization heuristic. Since there are 280 instances in total, the results are aggregated by the level of tightness of the

\footnotetext{
${ }^{6}$ To the best of our knowledge, these are the best known upper bounds.
} 
time lag constraints. For each set, we give the ratio of instances that were solved to optimality in at least one of the five runs in the first column, as well as the mean PRD in the second column.

First, we notice the great impact of the new initialization heuristic on our method. Without it, the Ilog Scheduler model was more efficient for instances with $y=1$, and the overall results are extremely poor for larger values of $y$. However, the mean results are deceptive. Without initialization, Model 3 can be very efficient, although in a few cases no solution at all can be found. Indeed, relaxing the makespan does not necessarily makes the problem easy for this model. The weight of these bad cases in the mean value can be important, hence the poor PRD. On the other hand, we can see that the Ilog Scheduler model is more robust to this phenomenon: a non-trivial upper bound is found in every case. It is therefore likely that the impact of the initialization heuristic will not be as important on the Ilog model as on Model 3.

We also notice that solution guidance and geometric restarts greatly improve Ilog Scheduler's performance. Interestingly, we observe that our approach is best when the time lag constraints are tight. On the other hand, Scheduler is slightly more efficient on instances with loose time lag constraints and in particular proves optimality more often on these instances. However, whereas our method always finds near-optimal solutions (the worst mean PRD is 0.47 for instances with $y=1$ ), both scheduler models find relatively poor upper bounds for small values of $y$.

\subsection{Job Shop Scheduling Problem with no wait constraints}

For the no-wait job shop problem, the best methods are a tabu search method by Schuster (TS [20]) and a hybrid constructive/tabu search algorithm introduced by Bożejko and Makuchowski in 2009 (HTS [6]). We also report the results of a Branch \& Bound procedure introduced by Mascis and Pacciarelli [17]. This algorithm was run on a Pentium II $350 \mathrm{MHz}$.

Table 5: NW-JSP - Comparison with related work (Upper bound PRD).

\begin{tabular}{|c|c|c|c|c|c|c|c|c|}
\hline \multirow{2}{*}{$\frac{\text { Instance }}{\text { la[1-10] }}$} & \multirow{2}{*}{\begin{tabular}{|c|} 
Mascis et al. \\
B\&B
\end{tabular}} & $\begin{array}{c}\text { Schuster } \\
\text { TS }\end{array}$ & \multicolumn{2}{|c|}{ Bożejko et al. } & \multicolumn{2}{|c|}{$\begin{array}{c}\text { Model } 4 \\
\text { tdom }+ \text { bw tdom } / t w\end{array}$} & \multicolumn{2}{|c|}{$\begin{array}{c}\text { Model 5 } \\
\text { tdom+bw tdom } / t w\end{array}$} \\
\hline & & 4.43 & 1.77 & 1.77 & $\begin{array}{l}0.00 \\
\end{array}$ & 0.00 & $\mathbf{0 . 0 0}$ & 0.00 \\
\hline $1 \mathrm{a}[11-20$ ] & 31.66 & 7.93 & 3.49 & 0.95 & 0.14 & 0.10 & 0.00 & 0.31 \\
\hline la [21-30] & 61.09 & 10.43 & 7.25 & 0.08 & 1.16 & 0.57 & 0.25 & 0.84 \\
\hline $1 \mathrm{a}[31-40]$ & 73.73 & 10.95 & 8.33 & 0.15 & 4.42 & 1.77 & 2.68 & 1.36 \\
\hline $\mathrm{abz}[5-9]$ & 47.04 & 9.01 & 5.95 & 0.78 & 2.47 & 1.14 & 1.13 & 1.20 \\
\hline orb [1-10 ] & 0.00 & 2.42 & 0.77 & 0.77 & 0.00 & 0.00 & 0.00 & 0.00 \\
\hline SWV [1-5] & 60.85 & 3.94 & 3.67 & 0.00 & 2.54 & 0.77 & 0.00 & 0.43 \\
\hline $\operatorname{sWV}[6-10]$ & 57.82 & 4.99 & 4.19 & 0.00 & 4.78 & 1.71 & 0.44 & 1.00 \\
\hline SWV [11-15] & 70.98 & 0.68 & 2.48 & 0.60 & 19.50 & 6.53 & 17.54 & 5.18 \\
\hline SwV [16-20] & 76.81 & 5.71 & 3.98 & 0.00 & 10.92 & 68.94 & 4.47 & 3.17 \\
\hline$y n[1-4]$ & 72.74 & 12.40 & 8.85 & 0.32 & 5.60 & 5.75 & 2.37 & 2.88 \\
\hline overall & 44.72 & 6.51 & 4.36 & 0.52 & 3.53 & 5.50 & 1.97 & 1.13 \\
\hline
\end{tabular}

For the no-wait class we used the same data sets as Schuster [20] and Bożejko et al. [6] where null time lags are added to instances of the OR-library. We report the best results of each paper in terms of average PRD. It should be noted that for HTS, the authors reported two sets of results. The former were run with a time limit based on the runtimes reported in [20] and 
varying from 0.25 seconds for the easiest instances to 2360 seconds for the hardest. The latter (in italic font, and referred to as HTS+ in Table 5) were run "without limit of computation time". We use bold face to mark the best result amongst methods that had time limits, i.e. excluding HTS+. We ran two variable ordering heuristics for our method. First, the heuristics used for ETJSP and TL-JSP, where the Boolean variable minimizing the value of $\left(\max \left(t_{i}\right)+\max \left(t_{j}\right)-\right.$ $\left.\min \left(t_{i}\right)-\min \left(t_{j}\right)+2\right) /\left(w\left(t_{i}\right)+w\left(t_{j}\right)\right)$ is chosen first, denoted $t d o m / t w$. Second, we used another heuristic, denoted $t d o m+b w$ that selects the next Boolean variable to branch on solely according to the tasks' domain sizes $\left(\max \left(t_{i}\right)+\max \left(t_{j}\right)-\min \left(t_{i}\right)-\min \left(t_{j}\right)+2\right)$, and break ties with the Boolean variable's own weight $w\left(b_{i j}\right)$.

Table 6: NW-JSP - New best upper bounds and optimality proofs.

\begin{tabular}{|c|c|c|c|c|c|c|c|c|}
\hline \multirow[b]{2}{*}{ Instance } & \multirow[b]{2}{*}{ BKS } & \multirow{2}{*}{$\begin{array}{c}\text { Schuster } \\
\text { TS }\end{array}$} & \multicolumn{2}{|c|}{ Bożejko } & \multicolumn{2}{|c|}{ Model 4} & \multirow{2}{*}{\multicolumn{2}{|c|}{$\begin{array}{c}\text { Model } 5 \\
\text { tdom+bw tdom } / t w\end{array}$}} \\
\hline & & & HTS & HTS+ & tdom $+b w$ & $m / t w$ & & \\
\hline la11_0_0 & 2821 & 1737 & 1704 & 1621 & 1622 & 1619 & $1619 *$ & 1621 \\
\hline la13_0_0 & 2650 & 1701 & 1696 & 1580 & 1582 & 1590 & $1580 *$ & 1580 \\
\hline la14_0_0 & 2662 & 1771 & 1722 & 1610 & 1578 & 1578 & $1578 *$ & 1612 \\
\hline la15_0_0 & 2765 & 1808 & 1747 & 1686 & 1692 & 1679 & $1671 *$ & 1691 \\
\hline la26_0_0 & 4268 & 2664 & 2738 & 2506 & 2624 & 2511 & 2488 & 2540 \\
\hline la28_0_0 & 4478 & 2886 & 2741 & 2552 & 2640 & 2605 & 2546 & 2569 \\
\hline 1a30_0_0 & 4097 & 2939 & 2791 & 2452 & 2452 & 2452 & $2452 *$ & 2508 \\
\hline la34_0_0 & 6380 & 3957 & 3936 & 3659 & 3914 & 3693 & 3817 & 3657 \\
\hline 1a39_0_0 & 4295 & 2804 & 2725 & 2687 & 2660 & 2660 & $2660 *$ & 2660 \\
\hline swv01 & 3824 & 2396 & 2424 & 2318 & 2344 & 2343 & 2318* & 2333 \\
\hline swv02 & 3800 & 2492 & 2484 & 2417 & 2440 & 2418 & $2417 *$ & 2417 \\
\hline swv05 & 3836 & 2482 & 2489 & 2333 & 2433 & 2333 & $2333^{*}$ & 2333 \\
\hline yn2 & 4025 & 2705 & 2647 & 2370 & 2486 & 2603 & 2427 & 2353 \\
\hline yn4 & 4109 & 2705 & 2630 & 2513 & 2532 & 2573 & 2499 & 2582 \\
\hline
\end{tabular}

In Table 6 we report the results on no-wait instances for which we obtained new upper bounds (5 instances) or new proofs of optimality (9 instances), thanks to the model introduced here.

\section{Conclusions}

We have shown that the simple constraint programming approach introduced in [13] can be successfully adapted to handle the objective of minimizing the sum of earliness/tardiness costs. These problems have traditionally proven troublesome for CP approaches because of the weak propagation of the sum objective [8].

Then we introduced a new heuristic to find good initial solutions for job shop problems with maximal time lag constraints. The resulting method greatly improves over state of the art algorithms for this problem. However, as opposed to the other aspects of the method (adaptive variable heuristic, solution guided branching, restarts with nogood storage) this new initialization heuristic is dedicated to job shop problems with time lag constraints.

Finally, we showed that domain-specific information can also be used to improve our model for no-wait job shop scheduling problems, allowing us to provide several improved upper bounds and prove optimality in many cases. 


\section{References}

1. C. Artigues, M-J. Huguet, and P. Lopez. Generalized Disjunctive Constraint Propagation for Solving the Job Shop Problem with Time Lags. EAAI, 24(2):220 - 231, 2011.

2. P. Baptiste, M. Flamini, and F. Sourd. Lagrangian Bounds for Just-in-Time Job-shop Scheduling. Computers \& OR, 35(3):906-915, 2008.

3. J. C. Beck. Solution-Guided Multi-Point Constructive Search for Job Shop Scheduling. JAIR, 29:4977, 2007.

4. J. C. Beck and P. Refalo. A Hybrid Approach to Scheduling with Earliness and Tardiness Costs. Annals OR, 118(1-4):49-71, 2003.

5. F. Boussemart, F. Hemery, C. Lecoutre, and L. Sais. Boosting Systematic Search by Weighting Constraints. In ECAI, pages 482-486, 2004.

6. W. Bozejko and M. Makuchowski. A Fast Hybrid Tabu Search Algorithm for the No-wait Job Shop Problem. Computers \& Industrial Engineering, 56(4):1502-1509, 2009.

7. A. Caumond, P. Lacomme, and N. Tchernev. A Memetic Algorithm for the Job-shop with Time-lags. Computers \& OR, 35(7):2331-2356, 2008.

8. E. Danna and L. Perron. Structured vs. unstructured large neighborhood search: A case study on job-shop scheduling problems with earliness and tardiness costs. Technical report, ILOG, 2003.

9. E. Danna, E. Rothberg, and C. Le Pape. Integrating Mixed Integer Programming and Local Search: A Case Study on Job-Shop Scheduling Problems. In CPAIOR, 2003.

10. T. Feydy and P. J. Stuckey. Lazy Clause Generation Reengineered. In CP, pages 352-366, 2009.

11. D. Grimes. A Study of Adaptive Restarting Strategies for Solving Constraint Satisfaction Problems. In AICS, 2008.

12. D. Grimes and E. Hebrard. Job Shop Scheduling with Setup Times and Maximal Time-Lags: A Simple Constraint Programming Approach. In CPAIOR, pages 147-161, 2010.

13. D. Grimes, E. Hebrard, and A. Malapert. Closing the Open Shop: Contradicting Conventional Wisdom. In $C P^{\prime} 09$, pages 400-408, 2009.

14. J. Kelbel and Z. Hanzálek. Solving production scheduling with earliness/tardiness penalties by constraint programming. J. Intell. Manuf., 2010.

15. C. Lecoutre, L. Sais, S. Tabary, and V. Vidal. Nogood Recording from Restarts. In IJCAI, pages 131-136, 2007.

16. M. Luby, A. Sinclair, and D. Zuckerman. Optimal Speedup of Las Vegas Algorithms. In ISTCS, pages 128-133, 1993.

17. A. Mascis and D. Pacciarelli. Job-shop Scheduling with Blocking and No-wait Constraints. EJOR, 143(3):498-517, 2002.

18. T. E. Morton and D. W. Pentico. Heuristic Scheduling Systems. John Wiley and Sons, 1993.

19. C. Rajendran. A No-Wait Flowshop Scheduling Heuristic to Minimize Makespan. The Journal of the Operational Research Society, 45(4):472-478, 1994.

20. C. J. Schuster. No-wait Job Shop Scheduling: Tabu Search and Complexity of Problems. Math Meth Oper Res, 63:473-491, 2006.

21. A. Schutt, T. Feydy, P. J. Stuckey, and M. Wallace. Why Cumulative Decomposition Is Not as Bad as It Sounds. In $C P^{\prime}$ 09, pages 746-761, 2009.

22. N. Tamura, A. Taga, S. Kitagawa, and M. Banbara. Compiling Finite Linear CSP into SAT. In $C P$, pages 590-603, 2006.

23. M. Vázquez and L. D. Whitley. A comparison of genetic algorithms for the dynamic job shop scheduling problem. In GECCO, pages 1011-, 2000.

24. P. Vilím. Filtering Algorithms for the Unary Resource Constraint. Archives of Control Sciences, 18(2), 2008.

25. T. Walsh. Search in a Small World. In IJCAI, pages 1172-1177, 1999.

26. J-P. Watson, L. Barbulescu, A. E. Howe, and L. D. Whitley. Algorithm performance and problem structure for flow-shop scheduling. In AAAI, pages 688-695, 1999.

27. D. A. Wismer. Solution of the Flowshop-Scheduling Problem with No Intermediate Queues. Operations Research, 20(3):689-697, 1972. 